\title{
Enhancement of immunogenicity and efficacy of a plasmid DNA rabies vaccine by nanoformulation with a fourth-generation amine-terminated poly(ether imine) dendrimer
}

\author{
This article was published in the following Dove Press journal: \\ International Journal of Nanomedicine \\ 28 January 2014 \\ Number of times this article has been viewed
}

\section{Padinjaremattathil \\ Thankappan Ullas' \\ Shampur Narayan \\ Madhusudana ${ }^{2}$ \\ Anita Desai ${ }^{2}$ \\ Bhadravathi Kenchappa \\ Chandrasekhar Sagar ${ }^{3}$ \\ Govindasamy Jayamurugan ${ }^{4}$ \\ Yamajala Bhaskara Rama \\ Durga Rajesh ${ }^{4}$ \\ Narayanaswami Jayaraman ${ }^{4}$}

'School of Chemical and

Biotechnology, Shanmugha Arts,

Science, Technology and Research

Academy (SASTRA) University,

Thanjavur, India; ${ }^{2}$ Department of

Neurovirology, ${ }^{3}$ Department of Neuropathology, National Institute of Mental Health and Neurosciences, Bangalore, India; ${ }^{4}$ Department of Organic Chemistry, Indian Institute of Science, Bangalore, India
Correspondence: Shampur Narayan Madhusudana

Department of Neurovirology,

NIMHANS, Hosur Road,

Bangalore, 560029 India

Tel +9l 8026995129

Fax +9l 8026564830

Email mshampur@gmail.com
Purpose: Delayed onset of, and low magnitude of, protective immune responses are major drawbacks limiting the practical utility of plasmid vaccination against rabies. In this study we evaluated whether nanoformulation with the novel poly(ether imine) (PETIM) dendrimer can enhance the immunogenicity and efficacy of a plasmid-based rabies vaccine.

Materials and methods: A plasmid vaccine construct (pIRES-Rgp) was prepared by cloning the full-length rabies virus glycoprotein gene into pIRES vector. Drawing upon the results of our previous study, a dendriplex (dendrimer-DNA complex) of pIRES-Rgp was made with PETIM dendrimer (10:1 w/w, PETIM:pIRES-Rgp). In vitro transfection was done on baby hamster kidney (BHK)-21 cells to evaluate expression of glycoprotein gene from pIRES-Rgp and PETIM-pIRES-Rgp. Subsequently, groups of Swiss albino mice were immunized intramuscularly with pIRES-Rgp or PETIM-pIRES-Rgp. A commercially available cell culture rabies vaccine was included for comparison. Rabies virus neutralizing antibody (RVNA) titers in the immune sera were evaluated on days 14, 28, and 90 by rapid fluorescent focus inhibition test. Finally, an intracerebral challenge study using a challenge virus standard strain of rabies virus was done to evaluate the protective efficacy of the formulations.

Results: Protective levels of RVNA titer ( $\geq 0.5 \mathrm{IU} / \mathrm{mL}$ ) were observed by day 14 in animals immunized with pIRES-Rgp and its dendriplex. Notably, PETIM-pIRES-Rgp produced 4.5-fold higher RVNA titers compared to pIRES-Rgp at this time point. All mice immunized with the PETIM-pIRES-Rgp survived the intracerebral rabies virus challenge, compared with $60 \%$ in the group which received pIRES-Rgp.

Conclusion: Our results suggest that nanoformulation with PETIM dendrimer can produce an earlier onset of a high-titered protective antibody response to a plasmid-based rabies vaccine. PETIM dendriplexing appears to be an efficacious nonviral delivery strategy to enhance genetic vaccination

Keywords: nonviral gene delivery, rabies prophylaxis

\section{Introduction}

Despite the availability of efficacious vaccines, rabies remains one of the most important zoonoses, with an estimated annual global mortality of around 50,000-70,000 in humans ${ }^{1}$ and much higher in the livestock. The disease, manifesting as a progressive fatal encephalomyelitis, is caused by ribonucleic acid (RNA) viruses of the genus Lyssavirus in the family Rhabdoviridae. The existence of huge numbers of diverse sylvatic animal reservoirs almost precludes the possibility of rabies eradication. However, human infections are entirely preventable by timely prophylaxis and efficacious 
vaccination of companion animals (especially dogs) as the major vectors of the disease in Asia and Africa. ${ }^{2}$ Vaccination of $70 \%$ of the canine population can drastically reduce human rabies in developing countries. ${ }^{3}$ However, this faces several challenges - difficult access to potent cell culture vaccines (costly and requiring cold chain and annual boosters), logistic issues, and suboptimal immune responses in animals in field conditions, to cite a few. ${ }^{3}$

Plasmid-based vaccination has been explored as an alternative strategy to cell culture-based rabies vaccines for animal prophylaxis., ${ }^{4,5}$ Deoxyribonucleic acid (DNA) vaccines are cheaper and easy to develop, stable at ambient temperature, able to induce prolonged cellular and humoral immune responses, and suitable for vaccination of pups. However, despite their demonstrated feasibility in several animal models, inefficient cellular delivery and poor immunogenicity remain major drawbacks, restricting their applicability in field-level immunization of animals. Much of the current research to improve the efficacy of plasmid vaccines is focused on two areas: molecular adjuvanting and novel delivery systems. ${ }^{6-11}$

A group of nanosized polymeric molecules called dendrimers has recently been reported to be a promising candidate in drug and gene delivery applications. These are globular, nanosized, hyperbranched polymeric molecules with precise molecular architecture and highly adaptable surface chemistries. ${ }^{12}$ The most well-known and commercially available dendrimers include the poly(amido amine) and poly(propylene imine) groups. Poly(ether imine) (PETIM) dendrimer, a novel dendrimer developed at the Indian Institute of Science, Bangalore, India, was reported to have a low cytotoxicity, and we have earlier reported its utility as an in vitro gene delivery vehicle. ${ }^{13-15}$

In this study we employed the plasmid construct pIRESRgp and its dendriplex with PETIM for immunization of Swiss albino mice and compared their immunogenicity and protective efficacy. Our preliminary findings indicate that PETIM-dendriplexing can enhance the systemic stability and cellular uptake of the complexed plasmid, thus favoring an enhanced immune response and increased efficacy.

\section{Materials and methods}

\section{Plasmids}

The bicistronic eukaryotic expression vector pIRES was a gracious gift from Dr Praveen K Gupta, Indian Veterinary Research Institute, Izatnagar, Uttar Pradesh, India. The plasmid pBacPak-GRC9 containing the full-length rabies virus glycoprotein of a street virus isolate (human) was earlier produced in the Department of Neurovirology, National Institute of Mental Health and Neurosciences (NIMHANS), Bangalore, India, as part of another project. EndoFree Plasmid Purification Giga Kit (QIAGEN, Düsseldorf, Germany) was used for the large-scale, endotoxin-free purification of the plasmid construct, as per manufacturer instructions.

\section{Enzymes and reagents}

TurboFect in vitro transfection reagent (catalog number R0531) and polymerase chain reaction reagents were obtained from Fermentas (Thermo Fischer Scientific, Waltham, MA, USA). The restriction enzymes NheI (catalog number R0131) and EcoRI (catalog number R0101S) were purchased from New England BioLabs Ltd. (Ipswich, MA, USA). The primers used in the study were designed using nucleotide sequences available in GenBank, using PRIMEGENS v2.0.5 software (Digital Biology Laboratory, University of MissouriColumbia, Columbia, MO, USA), and synthesized at Eurofins Genomics India Pvt Ltd., Bangalore, India.

\section{Antibodies and antibody conjugates}

The murine monoclonal antibody to rabies virus glycoprotein used in the study was produced earlier in the Department of Neurovirology, NIMHANS. ${ }^{16}$ Goat anti-mouse immunoglobulin $\mathrm{G}$ (IgG)-fluorescein isothiocyanate (FITC) (catalog number 621120380011730) was obtained from Merck Millipore (Billerica, MA, USA). The Second International Reference Serum obtained from National Institute for Biological Standards and Control, Hertfordshire, UK, was employed for calibration of an in-house reference serum used as standard in the rapid fluorescent focus inhibition test.

\section{Dendrimer}

A fourth-generation, amine-terminated PETIM dendrimer, bearing 32 peripheral amine groups (Figure 1) was synthesized and provided by Dr Narayanaswami Jayaraman at the Department of Organic Chemistry, Indian Institute of Science, Bangalore, India. The details of synthesis and physicochemical characterization of PETIM dendrimer have been reported previously. ${ }^{13-15}$

\section{Rabies virus and rabies vaccine}

The challenge virus standard (CVS)-11 strain of rabies virus used in the study was obtained as a lyophilized mouse brain homogenate from Central Research Institute, Kasauli, India and maintained by serial passage in suckling mice brains. Rabipur (PCEC vaccine manufactured by Chiron Behring Vaccines Pvt Ltd., Mumbai, India) vaccine (B No 1894) 


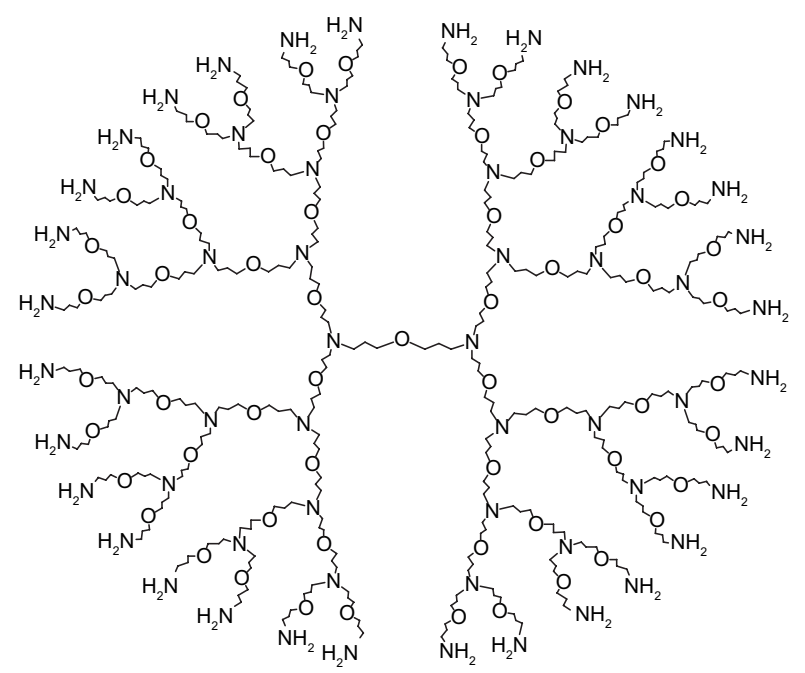

Figure I Diagram showing the structure of the fourth generation amine-terminated PETIM dendrimer employed in the study.

Note: Each molecule has 32 peripheral amine groups.

Abbreviation: PETIM, poly(ether imine).

having a potency $\geq 2.5 \mathrm{IU} / \mathrm{mL}$ was employed in the immunization study.

\section{Experimental animals}

Permission for conducting the animal experiments was obtained from the Institutional Animal Ethics Committee, NIMHANS. Four- to 6-week-old Swiss albino mice of either sex were used for the experiments. The guidelines of the Institutional Animal Ethics Committee were observed in the care of and experimentation on animals.

\section{Development of the plasmid constructs pIRES-Rgp}

The full-length rabies virus glycoprotein gene was amplified using Pfu DNA polymerase by Polymerase Chain Reaction, using pBacPak-GRC9 plasmid as template, and the gene-specific primers GFor (ATTAGCTAGCATGGTTCCTCAGGCTCTCC) and GRev (ATGTAGAATTCTCACAGTCTGGTCTGACC) containing NheI and EcoRI restriction sites (underlined), respectively. The glycoprotein gene was sub-cloned into the Multiple Cloning Site-A (MCSA) of the pIRES vector, using standard cloning protocols, yielding pIRES-Rgp.

\section{Preparation of PETIM-pIRES-RgP dendriplex}

Briefly, a 10:1 (w/w) (PETIM to pIRES-Rgp) was prepared by adding $900 \mu \mathrm{g}$ of PETIM (diluted from a stock solution of $25 \mathrm{mg} / \mathrm{mL}$ ) and $90 \mu \mathrm{g}$ of pIRES-Rgp in a total volume of $100 \mu \mathrm{L}$ of phosphate buffered saline (pH 7.4). The mixture was pulse-vortexed, briefly spun down, and incubated at room temperature for 20 minutes to facilitate dendriplex formation. A mixture prepared in parallel was subjected to electrophoresis in a $0.8 \%$ agarose gel $(100 \mathrm{~V}, 30$ minutes) to verify dendriplex formation as reported previously. ${ }^{15}$

\section{Analysis of dendriplex morphology by transmission electron microscopy}

The PETIM-pIRES-Rgp dendriplex was prepared as before, and stained by Harris' method. ${ }^{17}$ Thirty $\mu \mathrm{L}$ of the dendriplex solution was spotted onto formvar coated copper grids and dried. The grids were stained using $1 \%$ phosphotungstic acid for 1 minute, dried using filter paper, and examined under a Tecnai G2 Bio-Twin TEM (FEI, 5651 GG Eindhoven, the Netherlands), operating at an electron beam of $80 \mathrm{kV}$ and equipped with a Megaview III CCD Camera (Olympus Soft Imaging Solutions GmbH, Münster, Germany). Representative images were acquired and documented.

\section{Transient transfection to study glycoprotein gene expression}

A transient transfection experiment was performed on BHK-21 cells to verify expression of the glycoprotein gene from pIRES-Rgp complexed to a cationic lipid and to PETIM, separately. Briefly, BHK-21 were trypsinized from a confluent T-25 monolayer and seeded in a 24-well tissue culture plate $\left(1 \times 10^{6} /\right.$ well $)$ in $1 \mathrm{~mL}$ of growth medium and incubated in an atmosphere of $5 \% \mathrm{CO}_{2}$ in a $37^{\circ} \mathrm{C}$ incubator. Upon reaching a confluency of $60 \%-70 \%$, cells were transfected by the complexes of pIRES-Rgp prepared with TurboFect in vitro transfection reagent (Fermentas), or with PETIM. The transfection complexes were prepared in a total volume of $100 \mu \mathrm{L}$ of serum-free medium by adding $2 \mu \mathrm{g}$ of pIRES-Rgp to TurboFect in vitro transfection reagent as per manufacturer guidelines, or PETIM dendrimer $(20 \mu \mathrm{g})$. For transfection, the growth medium in the wells was replaced with $900 \mu \mathrm{L}$ of maintenance medium (with $1 \%$ fetal bovine serum), followed by dropwise addition of the mixtures and gentle rocking of the plate. After an incubation period of 30 minutes, the medium was aspirated off from each well and the monolayer rinsed in serum-free medium before replacement with $1 \mathrm{~mL}$ of complete growth medium. The plate was returned to $37^{\circ} \mathrm{C}$ and incubation continued.

At the end of 48 hours, the contents of the wells were aspirated off, and the transfected cells were fixed using chilled acetone. Expression of glycoprotein in the transfected cells was evaluated by indirect immunofluorescent staining. Briefly, the cell monolayer was incubated with $100 \mu \mathrm{L}$ of 
a murine monoclonal antibody against rabies glycoprotein (diluted 1:100 in phosphate buffered saline, $\mathrm{pH}$ 7.2) for 1 hour at $37^{\circ} \mathrm{C}$. After removing the unbound antibody by rinsing, $100 \mu \mathrm{L}$ of the secondary antibody (diluted 1:40 in phosphate buffered saline, $\mathrm{pH}$ 7.2) was added to the wells and the plate incubated in the dark for 30 minutes. The wells were rinsed again and finally examined under an inverted fluorescent microscope (NIKON Eclipse TS100, Nikon Instruments, Melville, NY, USA) for presence of specific fluorescence. Representative images were recorded using an attached digital camera.

\section{Evaluation of the immunogenicity and protective ability of pIRES-Rgp and its PETIM dendriplex}

Five groups of 4- to 6-week-old Swiss albino mice $(n=10)$ of either sex were immunized intramuscularly in the hind limb with the pIRES-Rgp alone (90 $\mu \mathrm{g}$ per animal) or PETIM-pIRES-Rgp dendriplex (10:1; w/w). The inocula were prepared in a total volume of $100 \mu \mathrm{L}$ of sterile normal saline. Three doses of the immunogens were administered on days 0,7 , and 21. Control groups were immunized with normal saline or empty pIRES vector. Additional groups of animals were also immunized with $100 \mu \mathrm{L}$ of Rabipur vaccine (1:100 dilution).

\section{Estimation of rabies virus neutralizing antibody titers}

Serum samples were collected from the immunized animals at day 14, 28, and 90 after the first immunization and their rabies virus neutralizing antibody (RVNA) titers were evaluated using a standard rapid fluorescent focus inhibition test with some modifications. ${ }^{18}$

\section{Evaluation of protective efficacy of the vaccine formulations}

A virus challenge study was performed 1 week after the third dose of vaccination, to evaluate the protective efficacy of the formulations. An intracerebral challenge dose of $50 \mathrm{LD}_{50}$ of CVS-11 strain of rabies virus was administered to all groups $(n=10)$ of immunized Swiss albino mice, and the percent survival recorded in each group after an observation period of 28 days.

\section{Statistical analysis}

The statistical analyses were done using GraphPad PRISM software (Version 5.00, San Diego, CA, USA). Comparison of antibody titers in the different groups was performed with nonparametric analysis of variance test, followed by post hoc analysis by Tukey's multiple comparison test. $P$-values $<0.05$ were considered to be statistically significant.

\section{Results}

\section{Development of the pIRES-Rgp construct}

The full-length rabies virus glycoprotein gene was amplified by polymerase chain reaction and successfully cloned into pIRES vector. The identity of the recombinant construct was verified by restriction enzyme analysis and nucleotide sequencing (data not shown).

\section{Studying PETIM-pIRES-Rgp morphology by transmission electron microscopy}

Analysis of the transmission electron microscopy (TEM) images revealed the PETIM-pIRES-Rgp dendriplex to be distributed primarily in toroidal nanoclusters having sizes of about $500 \mathrm{~nm}$. PETIM molecules, in comparison, appeared mostly in chains and aggregates of varying sizes (Figure 2).

\section{Preparation of PETIM-pIRES-RgP dendriplex and evaluation of glycoprotein gene expression}

PETIM-pIRES-Rgp dendriplex prepared at a ratio (w/w) of 10:1 was used for immunization, based on our earlier observation of successful transfection at this ratio, and also to keep the dendrimer concentration within nontoxic limits. The transfection ability of the dendriplex was tested initially on BHK-21 cells and expression of the encoded glycoprotein verified by indirect immunofluorescent staining using specific antibodies. As seen in Figure 3, typical membrane fluorescence suggestive of expression of glycoprotein was

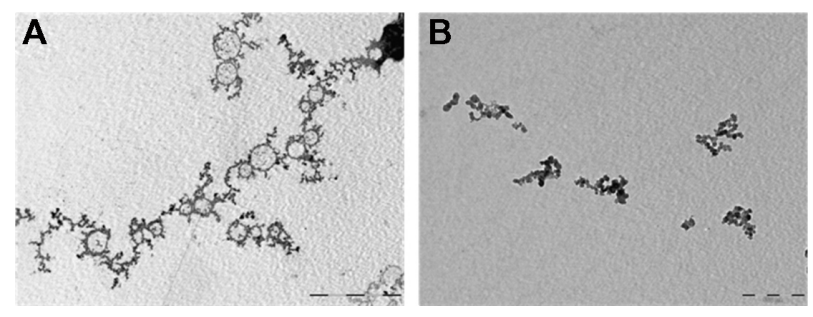

Figure 2 Transmission electron micrograph of PETIM and PETIM-pIRES-RgP dendriplex $(10: 1 ; w / w)$ used in the study.

Notes: Copper grids spotted with PETIM or PETIM-pIRES-RgP dendriplex were stained using $1 \%$ phosphotungstic acid, dried, and visualized under a Tecnai G2 Bio-Twin TEM (FEI, 565I GG Eindhoven, the Netherlands). Images show the TEM appearance of (A) PETIM dendrimer (I8,500×; scale bar: $5 \mu \mathrm{m})$ and (B) PETIMpIRES-Rgp dendriplex (30,000x; scale bar: $500 \mathrm{~nm}$ )

Abbreviations: PETIM, poly(ether imine); pIRES-Rgp, ; TEM, transmission electron microscope. 

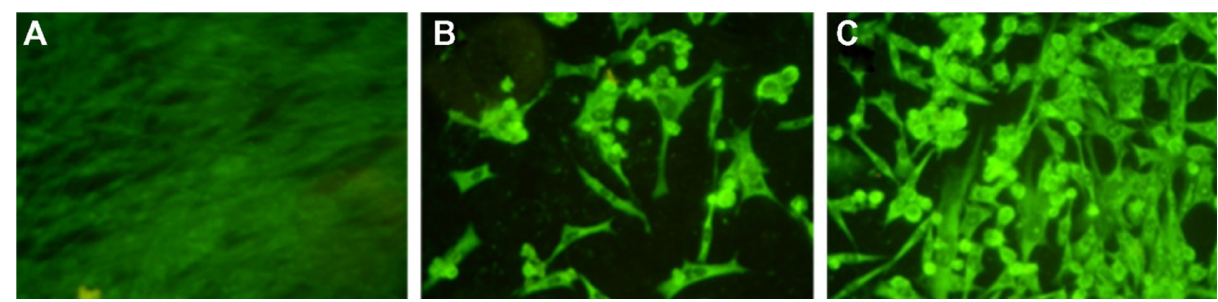

Figure 3 Images of BHK-2I cells fluorescently stained for glycoprotein expression.

Notes: BHK-2I cells were mock-transfected, or transfected with lipoplexed pIRES-RgP or PETIM-pIRES-RgP dendriplex, as described in the text. Forty-eight hours later, glycoprotein expression in the cells was evaluated by indirect immunofluorescent staining using a glycoprotein-specific murine primary antibody and FITC-labeled secondary IgG antibody. (A) Mock-transfected cell. (B) Cells transfected with lipoplexed pIRES-Rgp. (C) Cells transfected with PETIM-pIRES-Rgp dendriplex. Note the apple-green fluorescence indicative of glycoprotein expression. (Images at a total magnification of $400 \times$ ).

Abbreviations: BHK, baby hamster kidney; FITC, fluorescein isothiocyanate; lgG, immunoglobulin G; PETIM, poly(ether imine).

observed in cells transfected with the liposomal and PETIM complexes of pIRES-Rgp.

\section{Evaluation of immunogenicity and efficacy}

Analysis of immune sera revealed that adequate titers ( $>0.5 \mathrm{IU} / \mathrm{mL})$ of RVNA developed by day 14 , in animals immunized with pIRES-Rgp and PETIM-pIRES-Rgp. As seen in Table 1, PETIM-pIRES-Rgp produced a 4.5-fold higher RVNA titer compared to pIRES-Rgp at day 14 postimmunization (4.5 versus $1.0 \mathrm{IU} / \mathrm{mL}$, respectively; $P<0.01$ ). While there was a significant fall in RVNA titer by day 90 in the group receiving pIRES-Rgp, it remained elevated at all three time points in the PETIM-pIRES-Rgp group $(P<0.01)$. As expected, inoculation with Rabipur produced a much higher RVNA response which was also sustained at higher levels until day 90 (Table 1).

\section{Protective efficacy}

Survival of immunized mice following an intracerebral challenge dose of $50 \mathrm{LD}_{50}$ of CVS-11 strain was evaluated

Table I RVNA titers estimated by RFFIT in Swiss albino mice following three IM doses of the respective immunogens on days 0,7 , and 21

\begin{tabular}{lllll}
\hline $\begin{array}{l}\text { Group } \\
\text { number }\end{array}$ & Immunogen & \multicolumn{3}{l}{ Mean RVNA titer (IU/mL) (range) } \\
\cline { 3 - 5 } & & Day I4 & Day 28 & Day 90 \\
\hline I & PIRES & $<0.5$ & $<0.5$ & $<0.5$ \\
II & PIRES-RgP & 1.0 & 2.5 & 1.5 \\
& & $(0.5-1.5)$ & $(I .0-3.5)^{*}$ & $(0.5-2.0)$ \\
III & PETIM-pIRES- & 4.5 & 6.5 & 4.0 \\
& Rgp & $(3.5-6.5)^{* *}$ & $(2.5-7.8)^{* *}$ & $(2.5-6.5)^{* *}$ \\
IV & Rabipur & 8.0 & 12.5 & 8.5 \\
& & $(4.5-10.5)^{* *}$ & $(9.5-13.5)^{* *}$ & $(4.5-11.5)^{* *}$ \\
V & Normal saline & $<0.5$ & $<0.5$ & $<0.5$ \\
\hline
\end{tabular}

Notes: Data represent results from a single representative experiment on five groups of ten mice each. $* P<0.05 ; * * P<0.01$.

Abbreviations: IM, intramuscular; PETIM, poly(ether imine); RFFIT, rapid fluorescent focus inhibition test; RVNA, rabies virus neutralizing antibody. to assess the protective efficacy of the vaccine formulations. Animals in the control group (inoculated with normal saline) developed symptoms of rabies and died by day 6 after the challenge dose. As seen in Figure 4, a survival rate of $60 \%$ was observed in the group intramuscularly immunized with pIRES-Rgp. Notably, the PETIM-pIRES-Rgp dendriplex afforded $100 \%$ protection to the immunized animals $(P<0.01)$, identical to Rabipur-induced protection.

\section{Discussion}

Protection from rabies is commensurate with the presence of neutralizing antibodies targeted primarily at the viral glycoprotein, and an RVNA titer $\geq 0.5 \mathrm{IU} / \mathrm{mL}$ is generally considered as adequate for protection. ${ }^{19}$ Plasmid vaccination against rabies is quite feasible, as the rabies glycoprotein gene can be easily cloned into suitable vectors and delivered in vivo, to generate immune responses. However, the utility of this strategy remains restricted to experimental settings involving small animals, mainly due to low immunogenicity. Prior attempts to enhance its efficacy have mostly employed cationic lipidbased and genetic adjuvants, and delivery strategies such as gene gun and electroporation. ${ }^{9,10}$ A number of nonviral DNA delivery vehicles, in the form of cationic lipids and micro- and nanoparticles, have been evaluated for efficient delivery of DNA vaccines..$^{20}$ However, nonviral vectored approaches in plasmid vaccination against rabies have mostly been limited to cationic lipids. ${ }^{10,11}$ Whereas toxicity may restrict the in vivo use of cationic lipids, the methods, such as electroporation, would be too cumbersome for practical application. Herein we report PETIM dendrimer as a novel nanopolymeric delivery platform for a plasmid-based rabies vaccine.

Fourth-generation amine-terminated PETIM dendrimer is a newly developed globular nanopolymer with an approximate diameter of $3.5 \mathrm{~nm}$ and a low cytotoxicity profile. We have earlier reported the efficient DNA-binding and nuclease protection abilities, along with cellular uptake and transfection 


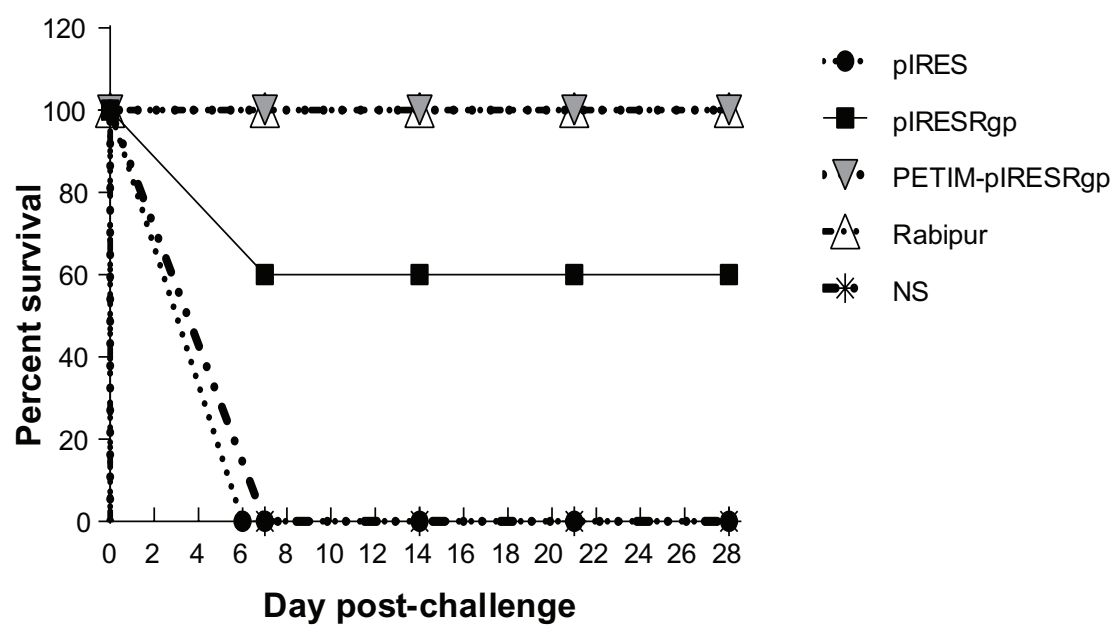

Figure 4 Graph showing survival rates in the immunized mice following intracerebral challenge with live rabies virus.

Notes: Data represent results from a single representative study on groups consisting of ten animals each. PETIM-pIRES-RgP and Rabipur were found to protect I00\% of the immunized mice against a subsequent lethal rabies virus challenge $(P<0.0 \mathrm{I})$.

Abbreviations: PETIM, poly(ether imine); NS, normal saline.

potential of this molecule, indicating its suitability as a gene delivery vehicle. ${ }^{15}$ The process of in vitro gene delivery using PETIM dendrimer has been patented by our group. ${ }^{21}$ Drawing upon the inferences from the earlier study, we hypothesized that complexation of a rabies vaccine plasmid with PETIM dendrimer might enhance its bioavailability, and hence, the ensuing immune responses as well.

Transmission electron microscopy revealed a toroidal morphology of the PETIM-pIRES-Rgp dendriplex, in agreement with previous reports on complexes of plasmid DNA with other dendrimers. However, the in vivo morphology of the complexes could be different from the ones seen by TEM, as sample preparation for TEM involves drying which might induce aggregation of the complexes and alterations in their morphology. ${ }^{22}$ The TEM images, however, yielded direct evidence of the ability of PETIM to complex with and condense pIRES-Rgp and an approximation of the dendriplex morphology in the dry state.

We used pIRES as the cloning vector in this study, as it was reported to induce protective RVNA titers in murine and canine models. ${ }^{23}$ Transient transfection of BHK-21 cells with pIRES-Rgp revealed robust expression of glycoprotein gene from its liposomal and dendrimer complexes (Figure 3), further verifying the in vitro gene delivery potential of PETIM.

Encouraged by these results, we extended our experiments to an animal model. Notably, pIRES-Rgp as well as PETIM-pIRES-Rgp dendriplex produced adequate RVNA titers $(>0.5 \mathrm{IU} / \mathrm{mL})$ in mice, 14 days after the first dose of each. This is in agreement with several previous reports that DNA-based rabies vaccines can indeed produce an early onset of protective RVNA titers. ${ }^{5,9}$ However, immunization with PETIM-pIRES-Rgp dendriplex induced a 4.5-fold higher RVNA titer compared to pIRES-Rgp. It may be likely that the nanorange size of the dendriplex $(\sim 500 \mathrm{~nm}$, as shown in Figure 2) facilitated their efficient uptake by the antigen presenting cells near the site of inoculation, leading to efficient antigen release and the generation of antibody responses. Considering the fact that the RVNA titers progressively fall in the absence of subsequent antigenic exposures, a higher level of RVNA titer produced following immunization might confer longer protection against rabies, before it trails off to levels below $0.5 \mathrm{IU} / \mathrm{mL}$. The present study, however, did not evaluate the duration of the protective antibody responses. The sustained high levels of RVNA titers on Day 90 in groups immunized with the PETIM-pIRES-Rgp dendriplex lend further evidence to the possibility of antigen retention and slow release by antigen-encapsulated nanospheres in the size range of 50-700 nm, as reported previously. ${ }^{24}$

In a recent study, Dutta et a ${ }^{25}$ reported the use of poly(propyleneimine) dendrimer and its dendrosomal formulation in genetic vaccination against hepatitis B. Following a single intramuscular injection of $10 \mu \mathrm{g}$ of an HBsAgencoding plasmid or its poly(propyleneimine) dendrimeric or its dendrosomal formulation, the highest level of total IgG anti-HBsAg antibodies was reported to be produced by the dendrosomal formulation. The immune responses peaked by $6-8$ weeks and displayed a Th1 bias. Another study by Balenga et $\mathrm{al}^{26}$ reported successful genetic vaccination against birch pollen allergy, mediated by Den123, a nontoxic selfassembled dendritic spheroidal nanoparticle made of biodegradable monomers. The authors reported a Th1/Th2 balanced 
immune response along with inhibition of immunoglobulin $\mathrm{E}$ response, probably resulting from a sustained release of the plasmid. The present study, however, achieved fairly good protective responses without additional modification of the PETIM-pIRES-Rgp dendriplex. Evaluation of IgG subtypes and cellular immune responses to the immunogens, however, were not attempted in the present study.

Ramya et al reported the use of poly(lactide-co-glycolide) microspheres to enhance immune responses to an inactivated rabies virus-based vaccine in a murine model. ${ }^{27}$ Oral and intraperitoneal administration of the microparticle-encapsulated immunogen yielded significantly higher antibody responses and protection compared to the unmodified vaccine. The authors suggested that the system could be a suitable platform for oral immunization against rabies. Though the present study did not attempt oral immunization, dendriplexing might offer a useful platform for noninvasive delivery of plasmid vaccines, and this needs to be evaluated further.

Though the pIRES-Rgp itself induced adequate RVNA response in mice by Day 14, it is surprising that only $60 \%$ protection was seen in this group compared to $100 \%$ in the group immunized with PETIM-pIRES-Rgp dendriplex. This is in agreement with Kaur et al, who reported variation in protective efficacy despite similar magnitude of immune responses. ${ }^{28}$ It is possible that cellular immune factors, in addition to RVNA, may also be involved in protection against intracerebral rabies virus challenge. The present study, however, did not evaluate the cellular immune responses to dendriplex vaccination.

Though the RVNA titers induced by PETIM-pIRESRgp were lesser compared to those produced by Rabipur, the protective efficacy of both was equal. It is worth mentioning here that the cell culture rabies vaccines require dedicated production facilities, are expensive, and demand cold chain maintenance and use within a specific time period after reconstitution, to retain their immunogenicity and efficacy. We assume that PETIM-pIRES-Rgp, in comparison, would be easier and cheaper to produce, more stable and tolerant of higher ambient storage temperatures, and thus represent a vaccine formulation suitable for lowresource settings.

To conclude, results from the present study indicate that nanoconjugation with the fourth-generation amineterminated PETIM dendrimer can enhance neutralizing antibody responses and protective efficacy to a plasmid-based rabies vaccine. This dendrimer might serve as a unique platform for the development of thermostable and efficacious genetic vaccines against a variety of zoonotic diseases, and needs further evaluation in this context.

\section{Acknowledgments}

The authors would like to thank the Indian Council of Medical Research for the grant of a Junior Research Fellowship to PTU.

\section{Disclosure}

The authors report no conflicts of interest in this work.

\section{References}

1. WHO Fact Sheet No 99 [webpage on the Internet]. WHO; 2010 [cited June 10, 2011]. Available from: http://www.paho.org/common/Display. asp?Lang=E\&RecID=10209. Accessed September 22, 2013.

2. Knobel DL, Cleaveland S, Coleman PG, et al. Re-evaluating the burden of rabies in Africa and Asia. Bull World Health Organ. 2005;83(5): 360-368.

3. Bahloul C, Taieb D, Diouani MF, et al. Field trials of a very potent rabies DNA vaccine which induced long lasting virus neutralizing antibodies and protection in dogs in experimental conditions. Vaccine. 2006;24(8):1063-1072.

4. Lodmell DL, Ray NB, Parnell MJ, et al. DNA immunization protects nonhuman primates against rabies virus. Nat Med. 1998;4(8):949-952.

5. Lodmell DL, Ray NB, Ulrich JT, Ewalt LC. DNA vaccination of mice against rabies virus: effects of the route of vaccination and the adjuvant monophosphoryl lipid A (MPL). Vaccine. 2000;18(11-12):1059-1066.

6. Lodmell DL, Parnell MJ, Bailey JR, Ewalt LC, Hanlon CA. Rabies DNA vaccination of non-human primates: post-exposure studies using gene gun methodology that accelerates induction of neutralizing antibody and enhances neutralizing antibody titers. Vaccine. 2002;20(17-18): 2221-2228.

7. Lodmell DL, Parnell MJ, Weyhrich JT, Ewalt LC. Canine rabies DNA vaccination: a single-dose intradermal injection into ear pinnae elicits elevated and persistent levels of neutralizing antibody. Vaccine. 2003;21(25-26):3998-4002.

8. Cupillard L, Juillard V, Latour S, et al. Impact of plasmid supercoiling on the efficacy of a rabies DNA vaccine to protect cats. Vaccine. 2005;23(16):1910-1916.

9. Fischer L, Minke J, Dufay N, Baudu P, Audonnet JC. Rabies DNA vaccine in the horse: strategies to improve serological responses. Vaccine. 2003;21(31):4593-4596.

10. Margalith M, Vilalta A. Sustained protective rabies neutralizing antibody titers after administration of cationic lipid-formulated pDNA vaccine. Genet Vaccines Ther. 2006;4:2.

11. Kaur M, Saxena A, Rai A, Bhatnagar R. Rabies DNA vaccine encoding lysosome-targeted glycoprotein supplemented with Emulsigen-D confers complete protection in preexposure and postexposure studies in BALB/c mice. FASEB J. 2010;24(1):173-183.

12. Astruc D, Boisselier E, Ornelas C. Dendrimers designed for functions: from physical, photophysical, and supramolecular properties to applications in sensing, catalysis, molecular electronics, photonics, and nanomedicine. Chem Rev. 2010;110(4):1857-1959.

13. Krishna TR, Jayaraman N. Synthesis of poly(propyl ether imine) dendrimers and evaluation of their cytotoxic properties. $J$ Org Chem. 2003;68(25):9694-9704.

14. Jayamurugan G, Jayaraman N. Synthesis of large generation poly(propyl ether imine) (PETIM) dendrimers. Tetrahedron. 2006;62(41): 9582-9588.

15. Thankappan UP, Madhusudana SN, Desai A, Jayamurugan G, Rajesh YB, Jayaraman N. Dendritic poly(ether imine) based gene delivery vector. Bioconjug Chem. 2011;22(2):115-119.

16. Muhamuda K, Madhusudana SN, Ravi V. Use of neutralizing murine monoclonal antibodies to rabies glycoprotein in passive immunotherapy against rabies. Hum Vaccin. 2007;3(5):192-195.

17. Harris JR. Negative staining of thinly spread biological samples. Methods Mol Biol. 2007;369:107-142. 
18. Smith JS, Yager PA, Baer GM. A rapid fluorescent focus inhibition (RFFIT) for determining rabies virus-neutralizing antibody. In: Meslin FX, Kaplan MM, Koprowsky H, editors. Laboratory Techniques in Rabies. 4th ed. Geneva: World Health Organization; 1996: 181-192.

19. WHO Expert Committee on Rabies. World Health Organ Tech Rep Ser. 1992;824:1-84.

20. Mintzer AM, Simanek EE. Nonviral vectors for gene delivery. Chem Rev. 2009;109(2):259-302.

21. Anita Desai et al, inventors; Poly(ether imine) dendrimers and uses thereof. Indian Patent. WO2012090223 A1. 2011, Dec 28.

22. Shcharbin D, Pedziwiatr E, Bryszewska M. How to study dendriplexes I: Characterization. J Control Release. 2009;135(3):186-197.

23. Patial S, Chaturvedi VK, Rai A, et al. Virus neutralizing antibody response in mice and dogs with a bicistronic DNA vaccine encoding rabies virus glycoprotein and canine parvovirus VP2. Vaccine. 2007;25(20):4020-4028.
24. Panyam J, Labhasetwar V. Biodegradable nanoparticles for drug and gene delivery to cells and tissue. Adv Drug Deliv Rev. 2003;55(3): 329-347.

25. Dutta T, Garg M, Jain NK. Poly(propyleneimine) dendrimer and dendrosome mediated genetic immunization against hepatitis B. Vaccine. 2008;26(27-28):3389-3394.

26. Balenga NA, Zahedifard F, Weiss R, Sarbolouki MN, Thalhamer J, Rafati S. Protective efficacy of dendrosomes as novel nano-sized adjuvants for DNA vaccination against birch pollen allergy. J Biotechnol. 2006;124:602-614.

27. Ramya R, Verma PC, Chaturvedi VK, et al. Poly(lactide-co-glycolide) microspheres: a potent oral delivery system to elicit systemic immune response against inactivated rabies virus. Vaccine. 2009;27(15): 2138-2143.

28. Kaur M, Rai A, Bhatnagar R. Rabies DNA vaccine: no impact of MHC class I and class II targeting sequences on immune response and protection against lethal challenge. Vaccine. 2009;27(15):2128-2137.
International Journal of Nanomedicine

\section{Publish your work in this journal}

The International Journal of Nanomedicine is an international, peerreviewed journal focusing on the application of nanotechnology in diagnostics, therapeutics, and drug delivery systems throughout the biomedical field. This journal is indexed on PubMed Central, MedLine, CAS, SciSearch $\AA$, Current Contents ${ }^{\circledR} /$ Clinical Medicine,

\section{Dovepress}

Journal Citation Reports/Science Edition, EMBase, Scopus and the Elsevier Bibliographic databases. The manuscript management system is completely online and includes a very quick and fair peer-review system, which is all easy to use. Visit http://www.dovepress.com/ testimonials.php to read real quotes from published authors. 